\title{
Integral Representations for Schwinger Functionals and the Moment Problem over Nuclear Spaces
}

\author{
H. J. Borchers and J. Yngvason \\ Institut für Theoretische Physik, Universität Göttingen, Göttingen, \\ Federal Republic of Germany \\ Received December 13, 1974
}

\begin{abstract}
It is shown that a continuous positive linear functional on a commutative nuclear *-algebra has an integral decomposition into characters if and only if the functional is strongly positive, i.e. positive on all positive polynomials. When applied to the symmetric tensor algebra over a nuclear test function space this gives a necessary and sufficient condition for the Schwinger functions of Euclidean quantum field theory to be the moments of a continuous cylinder measure on the dual space. Another application is to the problem of decomposing a Wightman functional into states having the cluster property.
\end{abstract}

\section{Introduction}

Whereas the extremal states of an abelian *-algebra of bounded operators on Hilbert space are at the same time the characters of the algebra, this is no longer true for algebras of unbounded operators ${ }^{1}$. In a previous article [2] an integral decomposition theory associated with the weak commutant of families of unbounded operators was used to obtain an extremal decomposition of states on nuclear *-algebras. The present paper is concerned with decompositions into characters in the commutative case. It is shown that such a decomposition is possible if and only if the state, satisfies a positivity condition which is well known from the classical moment problem over finite dimensional spaces $[3,4]$. This result can be applied to Euclidean quantum field theory where the sequence of Schwinger distributions defines by assumption a positive linear functional on the symmetric tensor algebra over some nuclear space of test functions. The condition tells also when a Wightman functional is an integral over states having the cluster property. That this is not always the case was shown in [2].

The infinite dimensional moment problem has been treated by several authors under conditions which at the same time guarantee the uniqueness of the solution, cf. e.g. [5] and [1]. Our method is based on the extension theory in [2] which, however, has to be modified slightly to fit our purpose. These changes are fairly straightforward so we can in most cases refer to [2] for the proofs. This method, which might appear somewhat indirect if one is only aiming at a solution of the moment problem (i.e. our Theorem 4.3$)^{2}$, has some advantages: It makes explicit the intimate connection of the solution with the weak commutant of the operators

1 See e.g. [1], Theorem 5.5.

2 After this research was completed a more direct proof of Theorem 4.3 was found by G. C. Hegerfeldt. A closely related result has also been obtained by M. Dubois-Violette (private communication). 
and the extension theory with subsidiary conditions developed in the next section is also useful for other purposes. In Section 5 we treat briefly the non-cyclic case where positivity has to be replaced by complete positivity in the sense of [6].

\section{Positive Extensions}

When not stated otherwise, the notations of this section are those of [2]. Let $(\mathscr{A}, \mathscr{D})$ be a ${ }^{*}$-operator family and assume the identity operator 1 belongs to $\mathscr{A}$. We shall be concerned with extensions $(\hat{\mathscr{A}}, \hat{\mathscr{D}})$ of $(\mathscr{A}, \mathscr{D})$ which preserve positivity of some of the operators in $\mathscr{A}$.

2.1. Definition. (i) An operator $A \in \mathscr{A}$ is called positive (on $\mathscr{D}$ ) if $\langle\varphi, A \varphi\rangle \geqq 0$ for all $\varphi \in \mathscr{D}$.

(ii) Let $\mathscr{P} \subset \mathscr{A}$ be some subset of positive operators on $\mathscr{D}$. An extension $(\hat{\mathscr{A}}, \hat{\mathscr{D}})$ is called $\mathscr{P}$-positive if $\hat{A}$ is positive on $\hat{\mathscr{D}}$ for all $A \in \mathscr{P}$. We denote the set $\{\hat{A} \mid A \in \mathscr{P}\}$ also by $\hat{\mathscr{P}}$.

2.2. Remarks. (i) If $\mathscr{P} C \mathscr{A}$ is a family of positive operators, then this holds also for the cone generated by the operators $B^{*} A B, B \in \mathscr{A}$ and $A \in \mathscr{P}$, whenever the product is defined.

(ii) Since an extension is assumed to preserve the algebraic structure of $\mathscr{A}$, every extension is positive with respect to the squares $A^{*} A$ when these are defined. More generally, a $\mathscr{P}$-positive extension is also positive w.r.t. the cone defined above.

(iii) The closure $(\overline{\mathscr{A}}, \overline{\mathscr{D}})$ of an operator family (cf. [2], 2.4) defines a $\mathscr{P}$-positive extension for any family $\mathscr{P} \subset \mathscr{A}$ of positive operators.

2.3. Definition. Let $\mathscr{P}$ be as above, and assume that $1 \in \mathscr{P}$. A bounded operator $x \in(\mathscr{A}, \mathscr{D})_{w}^{\prime}$ will be called strongly positive (w.r.t. $\mathscr{P}$ ) and we write $x \gg 0$, or sometimes $x \gg_{\mathscr{P}} 0$, if $x A$ is positive on $\mathscr{D}$ for all $A \in \mathscr{P}$. Instead of $x-y \gg 0$ we shall also write $y \ll x$.

In the following we shall assume that some fixed $\mathscr{P}$ with $1 \in \mathscr{P}$ is given and instead of " $\mathscr{P}$-strongly positive" etc. we shall simply write "strongly positive" etc.

2.4. Remark. (i) Because $1 \in \mathscr{P}$, every $x \gg 0$ is positive in the usual sense. The converse holds if $x$ commutes strongly with the closures $\bar{A}$ of $A \in \mathscr{P}$, because then we have $x A \subset x^{1 / 2} \bar{A} x^{1 / 2}$ for $x \geqq 0$. In general, however, the two concepts are different.

(ii) Every $x \gg 0$ is also strongly positive w.r.t. the cone defined in 2.2(i).

2.5. Notation. $\mathscr{C}_{1}^{+}:=\left\{x \in(\mathscr{A}, \mathscr{D})_{w}^{\prime} \mid 0 \ll x \ll 1\right\}$,

$$
\mathscr{C}_{1}:=\mathscr{C}_{1}^{+}-\mathscr{C}_{1}^{+} \text {. }
$$

If we want to stress the dependence on $\mathscr{A}, \mathscr{P}$, and $\mathscr{D}$ we write $\mathscr{C}_{1}^{+}(\mathscr{A}, \mathscr{P}, \mathscr{D})$ etc.

2.6. Lemma. (i) The sets $\mathscr{C}_{1}^{+}$and $\mathscr{C}_{1}$ are convex and weakly compact. (ii) Let $(\hat{A}, \hat{\mathscr{D}})$ be a $\mathscr{P}$-positive extension and define the mapping $\varrho$ as in $[2]$, i.e. $\varrho(x)=P \times P$ where $P$ is the projector $\mathscr{H}(\hat{D}) \rightarrow \mathscr{H}(\mathscr{D})$ and $x$ is a bounded operator on $\mathscr{H}(\hat{\mathscr{D}})$. Then we have that $x \gg_{\mathscr{P}} 0$ implies $\varrho(x) \gg 0$ so $\varrho$ maps $\mathscr{C}_{1}^{+}(\hat{\mathscr{A}}, \hat{\mathscr{P}}, \hat{\mathscr{D}})$ into $\mathscr{C}_{1}^{+}(\mathscr{A}, \mathscr{P}, \mathscr{D})$.

Proof. The statements follow immediately from the definition and the weak compactness of the order interval $0 \leqq x \leqq 1$. 
2.7. Definition. (i) An induced extension $(\hat{\mathscr{A}}, \hat{\mathscr{M}}, \hat{\mathscr{D}})$ of $(\mathscr{A}, \mathscr{D})$ (cf. [2], Definition 2.10) will be called regular positive (with respect to $\mathscr{P}$ ), if

(a) It is positive, i.e. $(\mathscr{A}, \hat{\mathscr{D}})$ is a positive extension of $(\mathscr{A}, \mathscr{D})$.

(b) $\varrho$ is one-to-one when restricted to $\mathscr{C}_{1}^{+}(\mathscr{\mathscr { A }} \vee \hat{\mathscr{M}}, \hat{\mathscr{P}}, \hat{\mathscr{D}})^{3}$.

(ii) We define a relation among $\mathscr{P}$-positive, resp. regular $\mathscr{P}$-positive induced extensions as follows:

$$
(\hat{\mathscr{A}}, \hat{\mathscr{M}}, \hat{\mathscr{D}})<(\hat{\mathscr{A}}, \hat{\hat{N}}, \hat{\mathscr{D}})
$$

if there is a subalgebra $\hat{\mathscr{M}} C \hat{\hat{\mathscr{N}}}$ such that $(\hat{\mathscr{A}} \vee \hat{\hat{\mathscr{M}}}, \hat{\hat{\mathscr{N}}}, \hat{\mathscr{D}})$ is a positive, resp. regular positive induced extension of $(\hat{\mathscr{A}} \vee \hat{\mathscr{M}}, \hat{D})$ with respect to $\hat{\mathscr{P}} \subset \hat{\mathscr{A}}$.

2.8. Lemma. (i) Let $\left(\hat{\mathscr{A}}^{-}, \hat{\mathscr{M}}^{-}, \hat{\mathscr{D}}^{-}\right)$be the closure of the induced extension $(\hat{\mathscr{A}}, \hat{\mathscr{M}}, \hat{\mathscr{D}})$, i.e. $\hat{\mathscr{M}}^{-}=$weak closure $\hat{\mathscr{M}}, \hat{\mathscr{D}}^{-}=$lin. span $\hat{\mathscr{M}}^{-} \hat{\mathscr{D}}$ and $\hat{\mathscr{A}}^{-}=$extension of $\hat{\mathscr{A}}$ to $\hat{\mathscr{D}}^{-}$. Then $\left(\hat{\mathscr{A}}^{-}, \hat{\mathscr{M}}^{-}, \hat{\mathscr{D}}^{-}\right)$is regular positive iff $(\hat{\mathscr{A}}, \hat{\mathscr{M}}, \hat{\mathscr{D}})$ is regular positive.

(ii) Let $(\hat{\mathscr{A}}, \hat{\mathscr{M}}, \hat{D})$ be a positive induced extension. Then the positive part of the unit ball $\hat{\mathscr{M}}_{1}^{+}$belongs to $\mathscr{C}_{1}^{+}(\hat{\mathscr{A}} \vee \hat{\mathscr{M}}, \hat{\mathscr{P}}, \hat{\mathscr{D}})$.

(iii) The relation $<$ is an order relation (i.e. transitive and reflexive) among regular positive extensions.

(iv) For every linearity ordered set of regular positive extensions there exists a regular positive extension which dominates all the extensions of the set.

Proof. (i) Since $\mathscr{C}_{1}^{+}\left(\hat{\mathscr{A}}^{-} \vee \hat{\mathscr{M}}^{-}, \hat{\mathscr{P}}^{-}, \hat{\mathscr{D}}^{-}\right) \subset \mathscr{C}_{1}^{+}(\hat{\mathscr{A}} \vee \hat{\mathscr{M}}, \hat{\mathscr{D}})$ the statement follows from the fact that an operator is positive iff its closure is positive.

(ii) Follows from (i) and Remark 2.4(i).

(iii) And (iv) are proved in the same way as Lemma 2.11. in [2].

2.9. Lemma. (1) Let $(\hat{\mathscr{A}}, \hat{\mathscr{M}}, \hat{\mathscr{D}})$ be a positive induced extension such that the restriction $\varrho 1 \hat{\mathscr{M}}$ is one-to-one. Define $\mathscr{M}:=\varrho(\hat{\mathscr{M}}), \mathscr{K}_{1}^{+}:=\varrho\left(\hat{\mathscr{M}}_{1}^{+}\right)$and $\phi: \mathscr{M} \times \mathscr{M} \rightarrow \mathscr{M}$ by the equation

$$
\phi\left(m_{1}, m_{2}\right)=\varrho\left(\varrho^{-1}\left(m_{1}\right) \cdot \varrho^{-1}\left(m_{2}\right)\right) .
$$

These objects have the following properties:

(i) $\mathscr{M}$ is the linear hull of $\mathscr{K}_{1}^{+}$.

(ii) $\mathscr{K}_{1}^{+}$is a convex subset of $\mathscr{C}_{1}^{+}(\mathscr{A}, \mathscr{D})$ with
(a) $1 \in \mathscr{K}_{1}^{+}$.
(b) $X \in \mathscr{K}_{1}^{+}$implies $1-x \in \mathscr{K}_{1}^{+}$.

(iii) The product $\phi$ on $\mathscr{M}$ is
(a) bilinear,
(b) associative,
(c) commutative,
(d) respects the involution,
(e) has 1 as a unit element,

and for all $m_{i} \in \mathscr{M}, k \in \mathscr{K}_{1}^{+}, A \in \mathscr{P}$ and $\varphi_{i} \in \mathscr{D}$ we have

(f) $\sum_{i j}\left\langle\varphi_{i}, \phi\left(m_{i}^{*}, k, m_{j}\right) A \varphi_{j}\right\rangle \geqq 0$.

${ }^{3} \hat{\mathscr{A}} \vee \hat{\mathscr{M}}$ denotes the algebra generated by $\hat{\mathscr{A}}$ and $\hat{\mathscr{M}}$, or, if $\hat{\mathscr{A}}$ is not an algebra, simply the set theoretical union of $\hat{\mathscr{A}}$ and $\hat{\mathscr{M}}$. 
(2) Conversely, suppose $\mathscr{M}, \mathscr{K}_{1}^{+}$, and $\phi$ are as in (i)-(iii). Then there exists a positive induced extension $(\hat{\mathscr{A}}, \hat{\mathscr{M}}, \hat{\mathscr{D}})$ such that $\varrho \uparrow \hat{\mathscr{M}}$ is injective, and

(a) $\mathscr{M}=\varrho(\hat{\mathscr{M}})$,

(b) $\phi\left(m_{1}, m_{2}\right)=\varrho\left(\varrho^{-1}\left(m_{1}\right) \cdot \varrho^{-1}\left(m_{2}\right)\right)$,

(c) $\varrho\left(\hat{\mathscr{M}}_{1}^{+}\right) \supset \mathscr{K}_{1}^{+}$.

This extension is uniquely determined by (a) and (b) up to unitary equivalence ${ }^{4}$. For two such extensions $\left(\hat{\mathscr{A}}^{1}, \hat{\mathscr{M}}^{1}, \hat{\mathscr{D}}^{1}\right)$ and $\left(\hat{\mathscr{A}}^{2}, \hat{\mathscr{M}}^{2}, \hat{\mathscr{D}}^{2}\right)$ associated with $\left(\mathscr{M}^{1}\right.$, $\left.\left(\mathscr{K}_{1}^{+}\right)^{1}, \phi^{1}\right)$ resp. $\left(\mathscr{M}^{2},\left(\mathscr{K}_{1}^{+}\right)^{2}, \phi^{2}\right)$ we have

$$
\left(\hat{\mathscr{A}}^{1}, \hat{\mathscr{M}}^{1}, \hat{\mathscr{D}}^{1}\right)<\left(\hat{\mathscr{A}}^{2}, \hat{\mathscr{M}}^{2}, \hat{\mathscr{D}}^{2}\right)
$$

if and only if $\mathscr{M}^{1} \subset \mathscr{M}^{2}$ and $\phi^{1}=\phi^{2} \uparrow \mathscr{M}^{1} \times \mathscr{M}^{1}$.

Proof. The verification of (1) is straightforward. Conversely, if we define $\mathscr{K}=\bigcup_{\lambda \geqq 0} \lambda \mathscr{K}_{1}^{+}$then the triple $(\mathscr{M}, \mathscr{K}, \phi)$ satisfies all the hypotheses of Lemma 2.12 in [2]. The $\mathscr{P}$-positivity of the extension follows immediately from (iii) (f).

2.10. Lemma. If $x \in \mathscr{C}_{1}^{+}, x \neq \lambda 1$ with $0<\lambda<1$, then

$$
\begin{aligned}
\mathscr{K}_{1}^{+} & :=\{\lambda x+\mu(1-x) \mid 0 \leqq \lambda \leqq 1,0 \leqq \mu \leqq 1\} \\
\mathscr{M} & :=\operatorname{lin} \cdot \operatorname{span} \mathscr{K}_{1}^{+}=\{\alpha x+\beta(1-x) \mid \alpha, \beta \in \mathbb{C}\}
\end{aligned}
$$

and

$$
\phi\left(\alpha_{1} x+\beta_{1}(1-x), \alpha_{2} x+\beta_{2}(1-x)\right):=\alpha_{1} \alpha_{2} x+\beta_{1} \beta_{2}(1-x)
$$

satisfy all hypotheses of Lemma 2.6. The algebra $\hat{\mathscr{M}}$ is generated by the identity and a projector $e$ with $\varrho(e)=x$.

Proof. If $\lambda x+\mu(1-x) \in \mathscr{K}_{1}^{+}$, then also $1-(\lambda x+\mu(1-x))=(1-\lambda) x+(1-\mu) \times$ $(1-x) \in \mathscr{K}_{1}^{+}$. Since $x \in \mathscr{C}_{1}^{+}$we have for all $A \in \mathscr{P}$ and $\lambda, \mu \geqq 0$ :

$$
\begin{aligned}
& \sum_{i j}\left\langle\varphi_{i}, \phi\left(\alpha_{i} x+\beta_{i}(1-x), \lambda x+\mu(1-x), \alpha_{j} x+\beta_{j}(1-x)\right) A \varphi_{j}\right\rangle \\
& =\lambda\left\langle\sum \alpha_{i} \varphi_{i}, x A \sum \alpha_{j} \varphi_{j}\right\rangle+\mu\left\langle\sum \beta_{i} \varphi_{i},(1-x) A \sum \beta_{j} \varphi_{j}\right\rangle \geqq 0 .
\end{aligned}
$$

The other properties are also easily verified.

2.11. Lemma. Let $x$ be as in the previous lemma. Define

$$
\begin{aligned}
\mathscr{C}_{x}^{+} & =\left\{y \in(\mathscr{A}, \mathscr{D})_{w}^{\prime} \mid 0 \ll y \ll x\right\} \\
\mathscr{C}_{x} & =\mathscr{C}_{x}^{+}-\mathscr{C}_{x}^{+}
\end{aligned}
$$

and $\mathscr{C}_{1-x}^{+}, \mathscr{C}_{1-x}$ in the same way. Then the extension defined by $x$ is regular positive if and only if

$$
\mathscr{C}_{x} \cap \mathscr{C}_{1-x}=\{0\} .
$$

Proof. The proof is a transcription of the proof of Lemma 2.15 in [2], replacing $\leqq$ by $\ll$.

2.12. Lemma. Let $x$ be as in Lemma 2.10. The extension defined by $x$ is regular positive if and only if $x$ is an extremal point of $\mathscr{C}_{1}^{+}(\mathscr{A}, \mathscr{P}, \mathscr{D})$.

${ }^{4}$ As in [2], unitary equivalence of two extensions $\left(\hat{\mathscr{A}}^{i}, \hat{\mathscr{M}}^{i}, \hat{\mathscr{D}}^{i}\right), i=1,2$ means there is a unitary operator $\mathscr{H}\left(\hat{\mathscr{D}}^{1}\right) \rightarrow \mathscr{H}\left(\hat{\mathscr{D}}^{2}\right)$ which reduces to the identity on $\mathscr{H}(\mathscr{D})$ and intertwines between $\mathscr{\mathscr { A }}^{1}$ and $\hat{\mathscr{A}}^{2}$ resp. $\hat{\mathscr{M}}^{1}$ and $\hat{\mathscr{M}}^{2}$. 
Proof. The statement follows from Lemma 2.11. in exactly the same way as Lemma 2.15 in [2] implies Lemma 2.14 in [2].

The next result is the counterpart to Theorem 2.16 and Lemma 3.1. in [2]:

2.13. Theorem. (i) Every regular positive extension is dominated by a maximal regular positive extension.

(ii) A regular positive extension $(\hat{\mathscr{A}}, \hat{\mathscr{M}}, \hat{\mathscr{D}})$ is maximal if and only if $\mathscr{C}_{1}^{+}(\hat{\mathscr{A}} \vee \hat{\mathscr{M}}, \hat{\mathscr{P}}, \hat{\mathscr{D}})=\hat{\mathscr{M}}_{1}^{+}$.

(iii) If $(\hat{\mathscr{A}}, \hat{\mathscr{M}}, \hat{\mathscr{D}})$ is a regular positive extension and if $\mathscr{H}(\mathscr{D})$ is separable, then $\mathscr{H}(\hat{\mathscr{D}})$ is also separable.

Proof. (i) follows from Lemmas 2.8 and 2.9 and Zorns lemma in the same way as in the proof of Theorem 2.16 in [2].

(ii) If $\mathscr{M}_{1}^{+}$is a proper subset of $\mathscr{C}_{1}^{+}$, there exists by the Krein-Mil'man theorem an extremal point $x \in \hat{\mathscr{C}}_{1}^{+}$with $X \notin \hat{\mathscr{M}}_{1}^{+}$. By Lemma 2.12 this $x$ defines a regular positive extension, which contradicts the maximality of $(\hat{\mathscr{A}}, \hat{\mathscr{M}}, \hat{\mathscr{D}})$.

(iii) The proof is the same as in Lemma 3.1 in [2].

\section{Standard Extensions for Commutative Algebras. The Cyclic Case}

Let $\mathfrak{A}$ be a commutative *-algebra with a unit element $I$. The concepts of an extension and $\mathscr{P}$-positivity, which up to now were used for operator families carry over to representations of $\mathfrak{A}$ in an obvious manner. We also recall a definition of Powers [1]: A representation $\Pi$ of $\mathfrak{A}$ on domain $\mathscr{D}$ is called standard if $\Pi(A)$ is essentially self-adjoint on $\mathscr{D}$ for all symmetric $A \in \mathfrak{U}$ and the spectral projectors for different operators commute.

Let $\mathscr{V} \subset \mathfrak{A}$ be some linear space of Hermitean generators for $\mathfrak{A}$, i.e. every element of $\mathfrak{A}$ is a polynomial $P\left(A_{1}, \ldots A_{n}\right)$ with $A_{i} \in \mathscr{V}$. If $\Pi$ is a standard representation of $\mathfrak{Q}$, then the spectrum of $\Pi\left(P\left(A_{1}, \ldots A_{n}\right)\right)$ belongs to the range of the polynomial $P\left(x_{1}, \ldots x_{n}\right)$ over $\mathbb{R}^{n}$, in particular, if $P\left(x_{1}, \ldots x_{n}\right)$ is a positive polynomial, then $\Pi\left(P\left(A_{1}, \ldots, A_{n}\right)\right)$ is a positive operator for all $A_{i} \in \mathfrak{U}$. To account for more general situations we introduce a notation.

3.1. Notation. If $\mathscr{Z}$ is any subset of the algebraic dual $\mathscr{V}^{*}$ we define

$$
\mathscr{P}(\mathscr{Z})=\left\{P\left(A_{1}, \ldots, A_{n}\right) \mid P\left(\omega\left(A_{1}\right), \ldots, \omega\left(A_{n}\right)\right) \geqq 0 \text { for all } \omega \in \mathscr{Z}\right\} .
$$

3.2. Theorem. Let $\Pi$ be a cyclic *-representation of $\mathfrak{A}$ with cyclic vector $\Omega$ and domain $\mathscr{D}=\Pi(\mathfrak{H}) \Omega$ and let $\mathscr{Z}$ and $\mathscr{P}(\mathscr{Z})$ be as above. The following conditions are equivalent.

(i) The functional $T(A)=\langle\Omega, \Pi(A) \Omega\rangle$ is positive on $\mathscr{P}(\mathscr{Z})$.

(ii) The representation $\Pi$ is $\mathscr{P}(\mathscr{Z})$-positive and if $(\hat{\Pi}, \hat{\mathscr{M}}, \hat{\mathscr{D}})$ is a maximal regular $\mathscr{P}(\mathscr{Z})$-positive extension then

(a) $\hat{\mathscr{M}} \Omega$ is dense in $\mathscr{H}(\hat{\mathscr{D}})$, so $\hat{\mathscr{M}}$ is maximal abelian.

(b) $\hat{\Pi}$ is standard.

(c) The joint spectrum of $\hat{\Pi}\left(A_{1}\right), \ldots, \hat{\Pi}\left(A_{n}\right), A_{i} \in \mathscr{V}$ belongs to the closure of $\left\{\left(\omega\left(A_{1}\right), \ldots, \omega\left(A_{n}\right)\right) \mid \omega \in \mathscr{Z}\right\}$. 
Proof. That (ii) $\Rightarrow$ (i) follows from spectral theory. The proof of the other direction uses similar ideas as the proof of Theorem 7.3 in [1]. We consider the algebra $\mathscr{A}=\hat{\Pi}(\mathfrak{U}) \vee \hat{\mathscr{M}}$ which is generated by $\mathscr{V}+\hat{\mathscr{M}}_{h}$, where $\hat{\mathscr{M}}_{h}$ is the Hermitean part of $\hat{\mathscr{M}}$. For simplicity of notation we denote the elements of $\mathscr{V}+\hat{\mathscr{M}}_{h}$ by $A_{1}, A_{2} \ldots$ etc. rather than $\hat{\Pi}\left(A_{1}\right), \hat{m}_{1}$ etc. If $S$ is any finite diemsional subspace of $\mathscr{V}+\mathscr{M}_{h}$ we let $\mathscr{A}_{S}$ denote the algebra generated by this space and define $\mathscr{D}_{S}=$ $\mathscr{A}_{S} \Omega, \mathscr{H}_{S}=$ closure of $\mathscr{D}_{S}$ in $\mathscr{H}(\mathscr{D})$ and $\mathscr{P}_{S}=\Pi(\mathscr{P}(\mathscr{Z})) \cap \mathscr{A}_{S}$. If $S$ is spanned by $A_{1}, \ldots, A_{k} \in \mathscr{V}$ and $A_{k+1}, \ldots, A_{n} \in \hat{\mathscr{M}}_{h}$ we let $\mathscr{Z}_{S}$ denote the set in the dual $S^{\prime}$ defined by the restriction of $\mathscr{Z}$ to the linear span of $\left\{A_{1}, \ldots A_{k}\right\}$ and the spectrum of the bounded operators $A_{k+1}, \ldots A_{n}$. Let $S_{0}$ be some fixed subspace and $P_{0}=$ $P\left(A_{1}, \ldots A_{n}\right)$ some fixed Hermitean element in $\mathscr{A}_{S_{0}}$. If $S$ is a finite dimensional subspace containing $S_{0}$ we consider the set $\mathfrak{C}_{S}$ of bounded operators $C$ on $\mathscr{H}(\hat{\mathscr{D}})$ satisfying

$(\alpha)\|C\| \leqq 1$,

( $\beta) C \in \mathscr{C}_{1}^{+}\left(\mathscr{A}_{S}, \mathscr{P}_{S}, \mathscr{D}_{S}\right)$,

( $\gamma) C\left(P_{0}^{2}+1\right) \Omega=\Omega$.

$\mathfrak{C}_{S}$ is weakly closed and $S_{1} \subset S_{2}$ implies $\mathfrak{C}_{S_{2}} \subset \mathfrak{C}_{S_{1}}$. We contend that $\mathfrak{C}_{S}$ is not empty for any $S$. In fact, by the solution to the finite dimensional moment problem $[3,4]$, the operators on $\mathscr{A}_{S}$ can be extended to multiplication operators on an $L_{2}$-space $\tilde{\mathscr{H}}_{S}$ over the closure of $\mathscr{Z}_{S}$. If $\left(\tilde{P}_{0}^{2}+1\right)$ is the extension of $\left(P_{0}^{2}+1\right)$ then the inverse $\left(\tilde{P}_{0}^{2}+1\right)^{-1}$ exists as multiplication with the reciprocal function. If $E_{S}$ is the projector $\tilde{\mathscr{H}}_{S} \rightarrow \mathscr{H}_{S}$ we define an operator $C_{S}$ on $\mathscr{H}(\hat{\mathscr{D}})$ by

$$
C_{S}=\left\{\begin{array}{lll}
E_{S}\left(\tilde{P}_{0}^{2}+1\right)^{-1} & \text { on } & \mathscr{H}_{S} \\
0 & \text { on } & \mathscr{H}_{S}^{\perp} .
\end{array}\right.
$$

This operator satisfies $(\alpha)-(\gamma)$, so $\mathfrak{C}_{S} \neq \emptyset$. Since all $\mathfrak{C}_{S}$ are closed subsets of the weakly compact unit ball we have therefore $\bigcap_{S_{0} \subset S} \mathfrak{E}_{S} \neq \emptyset$. If $C \in \bigcap_{S_{0} \subset S} \mathfrak{E}_{S}$, then $C \in \mathscr{C}_{1}^{+}(\hat{\Pi}(\mathfrak{A}) \vee \hat{M}, \hat{\mathscr{P}}(\mathscr{Z}), \hat{\mathscr{D}})$, so by the maximality of the extension we have $C \in \mathscr{M}$ and therefore $C\left(P_{0}^{2}+1\right)=\left(P_{0}^{2}+1\right) C=I$ on $\mathscr{\mathscr { D }}$. Passing to the closure we have that $\overline{\left(P_{0}^{2}+1\right)}$ is the inverse of the bounded self-adjoint operator $C$, so $\left(P_{0}^{2}+1\right) \Omega=C^{-1} \Omega \in \overline{\mathscr{M}} \Omega$. Vectors of the Form $\left(P^{2}+1\right) \Omega$ span the whole of $\hat{\mathscr{D}}$, so $\overline{\mathscr{M}} \Omega=\mathscr{H}$ and $\hat{\mathscr{M}}$ is therefore maximal abelian. Every $\hat{\Pi}(A)$ commutes strongly with $\hat{\mathscr{M}}$, so the closure $\overline{\hat{\Pi}(A)}$ is affiliated with the abelian algebra $\hat{\mathscr{M}}=\hat{\mathscr{M}}^{\prime}$ and therefore self-adjoint if $A=A^{*}$. The spectral projectors belong also to $\hat{\mathscr{M}}$ so $\hat{\Pi}$ is standard. Finally if $\left(x_{1}, \ldots, x_{n}\right)$ is not in the closure of $\left\{\omega\left(A_{1}\right), \ldots, \omega\left(A_{n}\right) \mid \omega \in \mathscr{Z}\right\}$ then there is a polynomial $P\left(A_{1}, \ldots, A_{n}\right) \in \mathscr{P}(\mathscr{Z})$ with $P\left(y_{1}, \ldots, y_{n}\right)<0$ for all $\left(y_{1}, \ldots y_{n}\right)$ in some neighbourhood of $\left(x_{1}, \ldots x_{n}\right)$. If this neighbourhood has a nonvoid intersection with the joint spectrum, then $\left\langle\varphi, \hat{\Pi}\left(P\left(A_{1}, \ldots, A_{n}\right)\right) \varphi\right\rangle$ for some $\varphi$ in contradiction to $\mathscr{P}(\mathscr{Z})$-positivity of $\hat{\Pi}$.

\section{The Moment Problem}

In this section we consider the decomposition of a linear functional on $\mathfrak{A}$ into characters, i.e. functionals $T$ with $T\left(A^{*} \cdot B\right)=\overline{T(A)} \cdot T(B)$. (It is convenient to exclude the case $T=0$, so this equation implies $T(I)=1$.) The decomposition theory associated with the maximal abelian algebra $\hat{\mathscr{M}}$ of the previous section 
is particularly nice if $\mathfrak{A}$ carries a nuclear topology. We shall therefore in this section assume that $\mathfrak{A}$ is a commutative, nuclear *-algebra with unit element $I$, and that $\mathscr{V}$ is a subspace of Hermitean elements in $\mathfrak{U}$ such that the *algebra $\mathfrak{U}(\mathscr{V})$ generated by $\mathscr{V} \cup\{I\}$ is dense in $\mathfrak{A} . \mathscr{V}$ is a real nuclear space with topological dual $\mathscr{V}^{\prime}$. If $\mathscr{Z} \subset \mathscr{V}^{\prime}$ we define $\mathscr{P}(\mathscr{Z})$ as in 3.1.

4.1. Theorem. Let $\mathfrak{U}, \mathscr{Z}$ and $\mathscr{P}(\mathscr{Z})$ be as above. The following conditions are equivalent for a linear functional $T$ on $\mathfrak{A}$ :

(i) $T$ is positive on $\mathscr{P}(\mathscr{Z})$ and the seminorm $A \rightarrow T\left(A^{*} A\right)^{1 / 2}$ is continuous ${ }^{5}$.

(ii) $T$ has a weak integral decomposition

$$
T=\int_{\Lambda} T_{\lambda} d \mu_{\lambda}
$$

where $\left(\Lambda, d \mu_{\lambda}\right)$ is a standard measure space [8] with $\int_{\Lambda} d \mu<\infty$ ( $\Lambda$ can be taken as the unit interval $[0,1])$ such that for almost all $\lambda$ the following holds true:

(a) $T_{\lambda}$ is a character and the restriction $T_{\lambda} \backslash \mathscr{V}$ belongs to the weak closure of $\mathscr{Z}$.

(b) $\{A \in \mathscr{P}(\mathscr{Z}) \mid T(A)=0\} \subset\left\{A \in \mathscr{P}(\mathscr{Z}) \mid T_{\lambda}(A)=0\right\}$.

(c) There is a continuous seminorm $p$ on $\mathfrak{A}$ and a function $\mathscr{C} \in L_{2}(\Lambda, d \mu)$ such that

$$
\left|T_{\lambda}(A)\right| \leqq \mathscr{C}(\lambda) p(A) \quad \text { for all } A \in \mathfrak{A} .
$$

Proof. The proof of (ii) $\Rightarrow$ (i) is straightforward. For the other direction, let $\Pi$ be the cyclic representation defined by $T$ and let $(\hat{\Pi}, \hat{M}, \hat{\mathscr{D}})$ be a maximal $\mathscr{P}(\mathscr{Z})$ positive extension ${ }^{6}$. The decomposition is obtained in the same way as in [2], them 3.3: The maximal abelian v. Neumann algebra $\hat{\mathscr{M}}$ on the separable Hilbert space $\mathscr{H}(\hat{\mathscr{D}})$ is unitarily equivalent to the multiplication algebra $L_{\infty}(\Lambda, d \mu)$ on $L_{2}(\Lambda, d \mu)$, where $d \mu$ is a measure on the spectrum $\Lambda$ of $\hat{\mathscr{M}}$. We have thus an integral decomposition of $\mathscr{H}(\hat{\mathscr{D}})$ :

$$
\mathscr{H}(\hat{\mathscr{D}})=\int_{\Lambda}^{\oplus} \mathscr{H}(\lambda) d \mu_{\lambda}
$$

where $\mathscr{H}(\lambda)=\mathbb{C}$ for all $\lambda$. By the nuclear spectral theorem $[9,10]$ the "projections" $\mathscr{D} \rightarrow \mathscr{H}(\lambda)$ are given by continuous linear operators $E_{\lambda}$. Since $\Omega$ is cyclic for $\hat{\mathscr{M}}$ we have $E_{\lambda} \Omega \neq 0$ for almost all $\lambda$. For these $\lambda$ we define

$$
T_{\lambda}(A)=\frac{1}{\left|E_{\lambda} \Omega\right|}\left\langle E_{\lambda} \Omega, E_{\lambda} \Pi(A) \Omega\right\rangle
$$

and zero otherwise. By integrating with arbitrary positive functions in $L_{\infty}(\Lambda, d \mu)$ one shows in the same way as in the proof of Theorem $11 \mathrm{ch}$. VIII in [9] or thm. 3.3 in $[2]^{7}$ that $T_{\lambda}$ is positive on $\mathscr{P}(\mathscr{Z})$ and (b) holds for almost all $\lambda . T_{\lambda}$ is a character because $\mathscr{H}(\lambda)=\mathbb{C}$, and the estimate (c) is part of the nuclear-spectral theorem. Finally, if $T_{\lambda}$ is not in the weak closure of $\mathscr{Z}$, then for some $A_{1}, \ldots A_{n} \in \mathscr{V}$ we have that $\left(T_{\lambda}\left(A_{1}\right), \ldots T_{\lambda}\left(A_{n}\right)\right) \in \mathbb{R}^{n}$ is separated from $\left\{\left(\omega\left(A_{1}\right), \ldots, \omega\left(A_{n}\right)\right) \mid \omega \in \mathscr{Z}\right\}$ by a

${ }^{5}$ The latter condition holds for all continuous, positive functionals, e.g. if $\mathfrak{A}$ is barrelled, cf. [7] thm. 4.1 or [2] thm. 3.7.

${ }^{6}$ The results of the previous section apply strictly speaking to the subalgebra $\mathfrak{A}(\mathscr{V}) \subset \mathfrak{A}$ which, however, is dense by assumption, so all statements carry over to $\mathscr{Q}$ by continuity of the representation.

7 The assumption of [2] that $\mathfrak{A}$ is separable is in fact superflous. This is so because the mappings $E_{\lambda}$ are all continuous w.r.t. a common seminorm (cf. (c)) and the corresponding normed space is separable by nuclearity of $\mathfrak{A}$, cf. [11] prop. 3.1.6. 
neighbourhood. There exists then a polynomial $P\left(x_{1}, \ldots x_{n}\right)$ with $P\left(A_{1}, \ldots A_{n}\right) \in$ $\mathscr{P}(\mathscr{Z})$ and $P\left(T_{\lambda}\left(A_{1}\right), \ldots, T_{\lambda}\left(A_{n}\right)\right)=T_{\lambda}\left(P\left(A_{1}, \ldots, A_{n}\right)\right)<0$. Hence, $T_{\lambda} \uparrow \mathscr{V} \in \overline{\mathscr{Z}}$ iff $T_{\lambda}$ is positive on $\mathscr{P}(\mathscr{Z})$, and as stated above, this is the case for almost all $\lambda$.

We now want to discuss the case when $\mathfrak{A}$ is the symmetric tensor algebra $S(\mathfrak{B})$ over a nuclear space. There is in general more than one natural way of defining a topology on the tensor product (even for nuclear spaces), but our choice probably covers most of the interesting cases. Let $\mathscr{V}$ be a real nuclear space which is a strict inductive limit of some countable family of its subspaces:

$$
\mathscr{V}=\underline{\lim } \mathscr{V}_{v} .
$$

We define the completed $n$-th tensorial power of $\mathscr{V}$ as the inductive limit of the completed $\pi$-tensorial powers [11] of the $\mathscr{V}_{v}$ :

$$
\mathscr{V}^{\hat{\oplus} n}:=\underline{\lim }_{\longrightarrow} \mathscr{V}_{v} \hat{\otimes}_{\pi} \ldots \hat{\otimes}_{\pi} \mathscr{V}_{v} .
$$

The (completed) $\mathbb{C}$-tensor algebra over $\mathscr{V}$ is the direct sum

$$
\mathscr{V}=\bigoplus_{n=0}^{\infty}\left(\mathscr{V}^{\hat{\oplus} n}+i \mathscr{V}^{\hat{\oplus} n}\right)
$$

equipped with the locally convex direct sum topology. The symmetric $\mathbb{C}$-tensor algebra $S(\mathscr{V})$ is derived from $\mathscr{V}$ in a standard way. Since $\mathscr{V}$ is real there is a natural involution on $S(\mathscr{V})$, so $S(\mathscr{V})$ is a commutative, nuclear *-algebra with unit.

4.2. Lemma. (i) If $T$ is continuous and positive on $S(\mathscr{V})$, then the seminorm $A \mapsto T\left(A^{*} A\right)^{1 / 2}$ is continuous.

(ii) Let $\mathscr{Z} \subset \mathscr{V}^{\prime}$. The continuous characters on $S(\mathscr{V})$ which are positive on $\mathscr{P}(\mathscr{Z})$ are in a one-to-one correspondence to the weak closure $\overline{\mathscr{Z}}$ via the formula

$$
\begin{aligned}
& T\left(P\left(A_{1}, \ldots, A_{n}\right)\right)=P\left(\omega\left(A_{1}\right), \ldots, \omega\left(A_{n}\right)\right) \\
& A_{i} \in \mathscr{V}, \omega=T \uparrow \mathscr{V} \in \overline{\mathscr{Z}} .
\end{aligned}
$$

Proof. (i) By definition of the $\pi$-topology, the seminorm is continuous on any $\mathscr{V}_{v}^{\hat{\oplus} n}$ and be definition of the inductive limit therefore on whole $S(\mathscr{V})$.

(ii) If $\omega \in \overline{\mathscr{Z}}$, then the formula defines a $\mathscr{P}(\mathscr{Z})$-positive character on $S(\mathscr{V})$. $T$ is even continuous in the $\pi$-topology on $\mathscr{V} \otimes \ldots \otimes \mathscr{V}$ which is in general coarser than the topology defined above. Conversely, if $T$ is $\mathscr{P}(\mathscr{Z})$-positive one shows that $T\lceil\mathscr{V} \in \overline{\mathscr{Z}}$ as at the end of the proof of Theorem 4.1.

4.3. Theorem. Let $T$ be a linear functional on $S(\mathscr{V})$ and $\mathscr{Z} \subset \mathscr{V}^{\prime}$. The following conditions on $T$ are equivalent.

(i) $T$ is continuous and positive on $\mathscr{P}(\mathscr{Z})$ with $T(I)=1$.

(ii) $T\left(P\left(A_{1}, \ldots, A_{n}\right)\right)=\int_{\Lambda} P\left(\omega_{\lambda}\left(A_{1}\right), \ldots, \omega_{\lambda}\left(A_{n}\right)\right) d \mu_{\lambda}$ where $\left(\Lambda, d \mu_{\lambda}\right)$ is a standard measure space with $\int_{A} d \mu_{\lambda}=1$ and

(a) $\omega_{\lambda} \in \overline{\mathscr{Z}}$ for almost all $\lambda$.

(b) The mapping $\lambda \mapsto \omega_{\lambda}(A)$ is measurable for every $A \in \mathscr{V}$, and there is a positive function $C \in L_{2}(\Lambda, d \mu)$ such that for every positive integrer $n$

$$
\left|\omega_{\lambda}(A)\right| \leqq \mathscr{C}(\lambda)^{1 / n} p_{n}(A)
$$

with a continuous seminorm $p_{n}$ on $\mathscr{V}$. 
(iii) $T\left(P\left(A_{1}, \ldots, A_{n}\right)\right)=\int_{\mathscr{\mathscr { X }}} P\left(\omega\left(A_{1}\right), \ldots, \omega\left(A_{n}\right)\right) d v_{\omega}$

where $d v$ is a measure on the $\sigma$-algebra generated by the weakly closed sets in $\mathscr{V}^{\prime}$ and having the following continuity property:

For every polynomially bounded continuous function $f$ on $\mathbb{R}^{n}$ the integral

$\int_{\overline{\mathscr{X}}} f\left(\omega\left(A_{1}\right), \ldots, \omega\left(A_{n}\right)\right) d v_{\omega}$

exists and is jointly continuous in $A_{1}, \ldots, A_{n} \in \mathscr{V}$.

Proof. That (iii) resp. (ii) imply (i) is obvious ${ }^{8}$. For (i) $\Rightarrow$ (ii) we apply Theorem 4.1 and Lemma 4.2. (i) to $S(\mathscr{V})$. In order to get the estimate (ii) (b) for $\omega_{\lambda}$ we note that by Theorem 4.1. (ii) (c) we have

$$
\left|T_{\lambda}\left(A_{1} \otimes \ldots \otimes A_{n}\right)\right|=\left|\omega_{\lambda}\left(A_{1}\right) \ldots \omega_{\lambda}\left(A_{n}\right)\right| \leqq C(\lambda) p\left(A_{1} \otimes \ldots \otimes A_{n}\right)
$$

with $\mathscr{C} \in L_{2}, \mathscr{C}(\lambda) \geqq 0$ and $p$ a continuous seminorm on $\mathscr{V}^{\oplus n}$. When restricted to $\mathscr{V}_{v}^{\oplus n}$, we can take $p$ of the form $q_{n} \otimes_{\pi} \ldots \otimes_{\pi} q_{n}$, so

$$
\left|\omega_{\lambda}(A)\right| \leqq \mathscr{C}(\lambda)^{1 / n} q_{n}(A)
$$

on $\mathscr{V}_{v}$. By definition of the inductive limit this implies

(ii) (b) on whole $\mathscr{V}$.

The measure on $\mathscr{V}^{\prime}$ is defined as the image of the measure on $\Lambda$ under the map $F: \lambda \mapsto \omega_{\lambda}$, i.e. we define a $\sigma$-algebra $\sum_{\mathscr{V}^{\prime}}$ on $\mathscr{V}^{\prime}$ as

$$
\sum_{\mathscr{r}^{\prime}}=\left\{M \mid F^{-1}(M) \in \sum_{\Lambda}\right\}
$$

where $\sum_{\Lambda}$ is the $\sigma$-algebra on $\Lambda$, and

$$
v(M):=\mu\left(F^{-1}(M)\right)
$$

for $M \in \sum_{r^{\prime}}$. A function $f$ on $\mathscr{V}^{\prime}$ is then $v$-integrable if and only if

$$
f_{*}(\lambda):=f(F(\lambda))
$$

is $\mu$-integrable on $\Lambda$ in which case

$$
\int_{M} f(\omega) d v_{\omega}=\int_{F^{-1}(M)} f_{*}(\lambda) d \mu_{\lambda} .
$$

The $\sigma$-algebra $\sum_{\mathscr{r}^{\prime}}$ contains all weakly closed sets: If $\tau$ is weakly closed, then $\omega_{\lambda} \in \tau$ iff the character $T_{\lambda}$ defined by $\omega_{\lambda}$ is positive on $\mathscr{P}(\tau)$, according to Lemma 4.2. (i). All $T_{\lambda}$ are continuous w.r.t. a fixed seminorm $p$ on $S(\mathscr{V})$ and in this seminorm, $S(\mathscr{V})$ and therefore also $\mathscr{P}(\tau)$ is separable. For every $P \in \mathscr{P}(\tau)$ the set $\left\{\lambda \mid T_{\lambda}(p) \geqq 0\right\}$ is $\mu$-measurable and $\left\{\lambda \mid \omega_{\lambda} \in \tau\right\}$ is measurable as a countable intersection of such sets. But $\left\{\lambda \mid \omega_{\lambda} \in \tau\right\}$ is the pre-image of $\tau$ under the map $F$, so $\tau$ is $v$-measurable.

It remains only to check the continuity property of $d v$. By (ii) (b) it holds for all polynomials so we have only to consider bounded continuous functions $f$. Now, since

$$
\int \sum_{i=1}^{n}\left|\omega\left(A_{i}\right)\right|^{2} d v_{\omega}
$$

${ }^{8}$ We note that the continuity property in (iii) or (ii) (b) imply continuity of $T$ in the $\pi$-topology on $\mathscr{V} \otimes \ldots \otimes \mathscr{V}$. 
is continuous in $A_{1}, \ldots A_{n}$, there exists for every $\varepsilon_{1}, \varepsilon_{2}>0$ a neighbourhood of zero $\mathscr{U} \subset \mathscr{V}$ such that the set $M_{\varepsilon_{1}}=\left\{\left.\omega\left|\sum\right| \omega\left(A_{i}^{0}-A_{i}\right)\right|^{2}>\varepsilon_{1}\right\}$ has measure $<\varepsilon_{2}$ if $A_{i}^{0}-A_{i} \in \mathscr{U}$ for $i=1, \ldots n$. Since $f$ is continuous, it is uniformly continuous on every disc $\left\{\left(x_{1}, \ldots x_{n}\right)\left|\sum\right| x_{1}^{0}-\left.x_{1}\right|^{2} \leqq r\right\}$ for $r<\infty$. Given $\varepsilon>0$ we first choose $\mathscr{U}_{1}$ such that $v\left(M_{1}\right) \cdot \sup |f|<\frac{\varepsilon}{4}$ and then $\mathscr{U}_{2} \subset \mathscr{U}_{1}$ such that for $A_{i}^{0}-A_{i} \in \mathscr{U}_{2}$

$$
\left|f\left(\omega\left(A_{1}^{0}\right), \ldots, \omega\left(A_{n}^{0}\right)\right)-f\left(\omega\left(A_{1}\right), \ldots, \omega\left(A_{n}\right)\right)\right| \cdot v\left(\mathscr{V}^{\prime} \backslash M_{1}\right)<\frac{\varepsilon}{2} .
$$

Under these conditions

$$
\int\left|f\left(\omega\left(A_{1}^{0}\right), \ldots, \omega\left(A_{n}^{0}\right)\right)-f\left(\omega\left(A_{1}\right), \ldots, \omega\left(A_{n}\right)\right)\right| d v_{\omega}<\varepsilon
$$

which completes the proof of the theorem.

In the next section the set $\mathscr{Z} C \mathscr{V}^{\prime}$ will be the dual of a convex cone in $\mathscr{V}$. As a final remark in this section we give for this case a description of $\mathscr{P}(\mathscr{Z})$ which is somewhat more direct than the original definition 3.1. in that no explicit reference is made to the dual space $\mathscr{V}^{\prime}$.

4.4. Lemma. Let $Q$ be a subset of $\mathscr{V}$ and define $Q^{\prime+}=\left\{\omega \in \mathscr{V}^{\prime} \mid \omega(A) \geqq 0\right.$ for all $A \in Q\}$. Let $\mathscr{K}(Q)$ denote the closed convex cone in $\mathscr{V}$ generated by $Q$.

Then

$$
\begin{aligned}
\mathscr{P}\left(Q^{\prime+}\right)= & \left\{P\left(A_{1}, \ldots, A_{n}\right) \mid A_{i} \in \mathscr{V}, P \text { real },\right. \\
& P\left(x_{1}, \ldots, x_{n}\right) \geqq 0 \text { for all }\left(x_{1}, \ldots x_{n}\right) \text { such that } y_{1} A_{1}+\ldots+y_{n} A_{n} \in \mathscr{K}(Q) \\
& \text { implies } \left.x_{1} y_{1}+\ldots+x_{n} y_{n} \geqq 0\right\} .
\end{aligned}
$$

In particular,

$$
\mathscr{P}\left(\mathscr{V}^{\prime}\right)=\left\{P\left(A_{1}, \ldots, A_{n}\right) \mid A_{i} \in \mathscr{V}, P\left(x_{1}, \ldots, x_{n}\right) \geqq 0 \quad \text { for all } \quad\left(x_{1}, \ldots x_{n}\right) \in \mathbb{R}^{n}\right\} .
$$

Proof. The latter statement is a particular case of the former with $Q=\{0\}$, because we can always choose $A_{1}, \ldots, A_{n}$ linearily independent.

Suppose $P\left(A_{1}, \ldots, A_{n}\right) \in \mathscr{P}\left(Q^{\prime+}\right), A_{i} \in \mathscr{V}$ linearily independent, and let $S$ be the linear span of the $A_{i}$ 's. Assume $\left(x_{1}, \ldots, x_{n}\right) \in \mathbb{R}^{n}$ satisfies the stated condition. We define a linear functional $\omega$ on $S$ by $\omega\left(\sum_{i=1}^{n} y_{i} A_{i}\right)=\sum_{i=1}^{n} x_{i} y_{i}$ which is then positive on $\mathscr{K}(Q) \cap S$. By the bipolar theorem, the restrictions of the functionals in $Q^{\prime+}$ to $S$ is weakly dense in the dual cone of $\mathscr{K}(Q) \cap S$. Since $P$ is continuous on $\mathbb{R}^{n}$, this implies $P\left(x_{1}, \ldots, x_{n}\right)=P\left(\omega\left(A_{1}\right), \ldots, \omega\left(A_{n}\right)\right) \geqq 0$.

The other inclusion is clear because if $\omega \in Q^{\prime+}$, then $\omega$ is also positive on $\mathscr{K}(Q)$, so $y_{1} A_{1}+\ldots y_{n} A_{n} \in \mathscr{K}(Q)$ implies $\omega\left(A_{1}\right) y_{1}+\ldots+\omega\left(A_{n}\right) y_{n} \geqq 0$.

\section{The Non-Cyclic Case}

In this section we want to indicate how the preceeding arguments have to be modified if the representation does not have a cyclic vector. The generalized version of Theorem 3.2 will be proved with the aid of an extension theorem of Powers [6] which has to be used instead of the Hahn-Banach theorem in the cyclic case. We refer also to [6] for a more thorough discussion of the notions 
of positive matrices of operators and completely positive maps which occur in the sequel.

Let $(\mathscr{A}, \mathscr{D})$ be a $*$-operator family and let $M(\mathscr{A})$ denote the set of matrices $A_{i j}$ with $A_{i j} \in \mathscr{A}$ and all but finitely many $A_{i j}=0$.

5.1. Definition. (i) A matrix $\left\{A_{i j}\right\} \subset M(\mathscr{A})$ is called positive on $\mathscr{D}$ if $\sum_{i j}\left\langle\varphi_{i}, A_{i j} \varphi_{j}\right\rangle \geqq 0$ for all $\varphi_{i} \in \mathscr{D}$.

(ii) If $\mathscr{P}_{M} \subset \bar{M}(\mathscr{A})$ is some subset of positive matrices on $\mathscr{D}$ we call an extension $(\hat{A}, \hat{D}) \mathscr{P}_{M}$-positive if $\left\{\hat{A}_{i j}\right\}$ is positive on $\hat{\mathscr{D}}$ for all $A_{i j} \in \mathscr{P}_{M}$.

5.2. Definition. Suppose $\mathscr{P}_{M}$ contains the matrix $\left\{\delta_{i 1}, \delta_{j 1}, I\right\}$ where $I$ is the identity operator. We say that $x \in(\mathscr{A}, \mathscr{D})_{w}^{\prime}$ is completely strongly positive with respect to $\mathscr{P}_{M}$ if $\left\{x A_{i j}\right\}$ is positive for all $\left\{A_{i j}\right\} \in \mathscr{P}_{M}$. We write then also $x \gg 0$ or $x \gg 0$.

In analogy with notation 2.5 we define $\mathscr{C}_{1}^{+}\left(\mathscr{A}, \mathscr{P}_{M}, \mathscr{D}\right)$ as the set of all operators in the weak commutant such that $x$ and $1-x$ are completely strongly positive w.r.t. $\mathscr{P}_{M}$. An extension is then called regular if $\varrho$ is injective when restricted to $\mathscr{C}_{1}^{+}\left(\hat{\mathscr{A}} \vee \hat{\mathscr{M}}, \hat{\mathscr{P}}_{M}, \hat{\mathscr{D}}\right)$.

The results of Section 2 are easily adapted to thus new situation and by exactly the same methods as before one proves:

5.3. Theorem. (i) Every regular $\mathscr{P}_{M}$-positive extension is majorized by a maximal regular $\mathscr{P}_{M}$-positive extension.

(ii) A regular $\mathscr{P}_{M}$-positive extension $(\hat{\mathscr{A}}, \hat{\mathscr{M}}, \hat{\mathscr{D}})$ is maximal if and only if $\mathscr{C}_{1}^{+}\left(\hat{\mathscr{A}} \vee \hat{\mathscr{M}}, \hat{\mathscr{P}}_{M}, \hat{\mathscr{D}}\right)=\hat{\mathscr{M}}_{1}^{+}$.

(iii) If $(\hat{\mathscr{A}}, \hat{\mathscr{M}}, \hat{\mathscr{D}})$ is a regular positive extension of $(\mathscr{A}, \mathscr{D})$ and $\mathscr{H}(\mathscr{D})$ is separable, then $\mathscr{H}(\hat{D})$ is also separable.

As in Section 3 the concepts defined above for operator families carry over to representations of a $*$-algebra $\mathfrak{A}$ in an obvious fashion. From now on we suppose that $\mathfrak{U}$ is commutative and that $\mathscr{V}, \mathscr{V}^{*}$ and $\mathscr{Z}$ are as in Section 3.

5.4. Notation.

$\mathscr{P}_{M}(Z)=\left\{P_{i j}\left(A_{1}, \ldots A_{n}\right) \mid A_{i} \in \mathscr{V}, P_{i j}\left(\omega\left(A_{1}\right) \ldots \omega\left(A_{n}\right)\right)\right.$ pos. definite for all $\left.\omega \in \mathscr{Z}\right\}$.

5.5. Theorem. Let $\Pi$ be a $\mathscr{P}_{M}(\mathscr{Z})$-positive representation of $\mathfrak{U}$ and let $(\hat{\Pi}, \hat{\mathscr{M}}, \hat{\mathscr{D}})$ be a maximal regular $\mathscr{P}_{M}(Z)$-positive extension. Then

(a) $\hat{\mathscr{M}}$ is maximal abelian.

(b) $\hat{\Pi}$ is standard.

(c) The joint spectrum of $\hat{\Pi}\left(A_{1}\right) \ldots \hat{\Pi}\left(A_{n}\right), A_{i} \in \mathscr{V}$ belongs to the closure of $\left\{\left(\omega\left(A_{1}\right) \ldots \omega\left(A_{n}\right)\right), \omega \in \mathscr{Z}\right\}$.

Conversely, every standard extension with $(c)$ is $\mathscr{P}_{M}(\mathscr{Z})$-positive.

Proof. We introduce two commutative *-algebras:

$$
\tilde{\mathfrak{M}}:=\left\{f: \mathscr{V}^{*} \rightarrow \mathbb{C} \mid f(\omega)=F\left(\omega\left(A_{1}\right), \ldots, \omega\left(A_{n}\right)\right), A_{i} \in \mathscr{V}\right.
$$

$F$ polynomially bounded and continuous on $\left.\mathbb{R}^{n}\right\}$

and

$$
\mathfrak{M}:=\left\{p: \mathscr{V}^{*} \rightarrow \mathbb{C} \mid p(\omega)=P\left(\omega\left(A_{1}\right), \ldots, \omega\left(A_{n}\right)\right), A_{i} \in \mathscr{V} \quad P \text { a polynomial on } \mathbb{R}^{n}\right\},
$$


which is a subalgebra of $\tilde{\mathfrak{M}}$. We let $\mathscr{B}$ resp. $\tilde{\mathscr{B}}$ denote the algebraic tensor products $\mathfrak{M} \odot \hat{\mathscr{M}}$ resp. $\tilde{\mathfrak{M}} \odot \hat{\mathscr{M}}$. The representation $\hat{\Pi}$ of $\mathfrak{A}$ defines in an obvious way a representation of $\mathfrak{M}$ on $\hat{\mathscr{D}}$ and since $\hat{\Pi}(\mathfrak{R})$ commutes with $\hat{\mathscr{M}}$ we have in fact a representation of $\mathscr{B}$ on $\hat{\mathscr{D}}$ which we again call $\hat{\Pi}$, by abuse of notation. This representation of $\mathscr{B}$ is completely positive with respect to $Q_{M}:=\left\{\left\{P_{i j}\right\} \odot 1\right\} \subset M(\mathscr{B})$, where the $\left\{p_{i j}\right\}$ are such that $\left\{p_{i j}(\omega)\right\}$ is pos. definite for all $\omega \in \mathscr{Z}$, and we may even extend $Q_{M}$ to an algebraically admissible cone in the sense of [6] by multiplying the matrices in $Q_{M}$ from both sides with a matrix in $M(\mathscr{B})$ and its adjoint and taking convex combinations. In analogous way we define $\tilde{Q}_{M}=\left\{\left\{f_{i j}\right\} \odot 1\right\} \subset M(\mathscr{B})$ where $\left\{f_{i j}(\omega)\right\}$. pos. definite for all $\omega \in \mathscr{Z}$, and extend $\tilde{Q}_{M}$ to an algebraically adminible cone. By Powers' extension theorem [6] (it is easy to verify that the hypotheses of this theorem are fulfilled) we many extend $\hat{\Pi}$ to a $\tilde{Q}_{M}$-positive map $\phi$ of $\mathscr{B}$ into bilinear forms on $\mathscr{D}$. As in [6] we use the notation $\langle\varphi|\phi(f)| \psi\rangle$ for these bilinear forms. Suppose $f \in \tilde{\mathfrak{M}} \subset \tilde{\mathscr{B}}$ and $0 \leqq f \leqq 1$. By positivity of $\tilde{\phi}$ we have for all $A, B \in \mathscr{B}, \varphi, \psi \in \hat{\mathscr{D}}$ :

$$
\left|\left\langle\varphi\left|\tilde{\varphi}\left(A^{*} f B\right)\right| \psi\right\rangle\right| \leqq\left\langle\varphi\left|\tilde{\phi}\left(A^{*} A\right)\right| \varphi\right\rangle^{1 / 2}\left\langle\psi\left|\tilde{\phi}\left(B^{*} B\right)\right| \psi\right\rangle^{1 / 2}=\mid \hat{\Pi}(A) \varphi\|\cdot\| \hat{\Pi}(B) \psi \|
$$

and also, for $\left\{B_{i j}\right\} \in Q_{M}$ and $\varphi_{i} \in \hat{\mathscr{D}}$

$$
0 \leqq \sum_{i j}\left\langle\varphi_{i}\left|\tilde{\phi}\left(f B_{i j}\right)\right| \varphi_{j}\right\rangle \leqq \sum_{i j}\left\langle\varphi_{i}\left|\tilde{\phi}\left(B_{i j}\right)\right| \varphi_{j}\right\rangle .
$$

Therefore, we have a well defined operator

$$
b \in \mathscr{C}_{1}^{+}\left(\hat{\Pi}(B), Q_{M}, \hat{\mathscr{D}}\right)=\mathscr{C}_{1}^{+}\left(\hat{\mathscr{M}}(\mathfrak{H}) \vee \hat{\mathscr{M}}, \mathscr{P}_{M}(\mathscr{Z}), \hat{\mathscr{D}}\right)=\hat{\mathscr{M}}_{1}^{+}
$$

if we put

$$
\langle\Pi(A) \varphi, b \Pi(B) \psi\rangle=\left\langle\varphi\left|\tilde{\phi}\left(A^{*} f B\right)\right| \psi\right\rangle .
$$

Let $P\left(A_{1}, \ldots A_{n}\right), A_{i} \in \mathscr{V}$ be a Hermitean element of $\mathfrak{A}$. The function $p(\omega)=$ $\left.P(\omega)\left(A_{1}\right), \ldots \omega\left(A_{n}\right)\right)$ takes then only real values, so $f_{ \pm}(\omega)=(\mathrm{p}(\omega) \pm i)^{-1}$ is a bounded function in $\mathfrak{M}$. $f_{ \pm}$can be written as a linear combination of functions $g_{i}$ with $0 \leqq g_{i} \leqq 1$, so $f_{ \pm}$defines an operator in $\hat{\mathscr{M}}$. If follows that $\left.\hat{\Pi}\left(P\left(A_{1}\right), \ldots, A_{n}\right)\right) \pm i I$ has on $\hat{\mathscr{D}}$ an inverse in $\hat{\mathscr{M}}$ so $\hat{\Pi}\left(P\left(A_{1}, \ldots, A_{n}\right)\right)$ is essentially self-adjoint. $\hat{\mathscr{M}}$ commutes strongly with $\hat{\Pi}(\mathfrak{M})$ and therefore with all bounded functions of the self-adjoint operators. If $\hat{\mathcal{N}}$ is any commutative v. Neumann algebra commuting with all bounded functions of every operator in $\hat{\Pi}(\mathfrak{M}) \vee \hat{\mathscr{M}}$, then

$$
\hat{\mathscr{N}}_{1}^{+} \subset \mathscr{C}_{1}^{+}(\hat{\Pi}(\mathfrak{H}) \vee \hat{\mathscr{M}}, \hat{\mathscr{P}}, \hat{\mathscr{D}})
$$

so $\hat{\mathscr{M}}$ is maximal abelian by maximality of the extension. That conversely every standard extension with (c) is $\mathscr{P}_{M}$-positive follows from spectral theory.

5.6. Remark. Since theorem 5.6 is a generalization of theorem 3.2 we have just given an alternate proof of the latter theorem.

If $\mathfrak{A}$ is a nuclear *-algebra and $\Pi$ a continuous representation we obtain an integral decomposition by combining Theorem 5.5 with the nuclear spectral theorem:

5.7. Theorem. Let $\mathfrak{A}$ be a commutative, nuclear *-algebra, $\mathscr{V}$ and $\mathscr{Z} \mathcal{C}^{\prime}$ as in thm. 4.1 and $\mathscr{P}_{M}(\mathscr{Z})$ as above. Suppose $(\hat{\Pi}, \hat{M}, \hat{\mathscr{D}})$ is a maximal regular $\mathscr{P}_{M}(\mathscr{Z})$-positive extension of some strongly continuous $\mathscr{P}_{M}(\mathscr{Z})$-positive representation $(\Pi, \mathscr{D})$ 
of $\mathfrak{A}$ on a separable Hilbert space $\mathscr{H}(\mathscr{D})$. Then there is a standard measure space $(\Lambda, d \mu)$ such that $\hat{\mathscr{M}}$ is unitarily equivalent to the algebra of all bounded multiplication operators on $L_{2}(\Lambda, d \mu)$ and the $\hat{\Pi}(A)$ 's are equivalent to multiplication operators with measurable functions $\lambda \mapsto \Pi_{\lambda}(A)$ which satisfy the following conditions:

(a) There are disjoint measurable sets $\Lambda_{i}, i=1,2,3, \ldots$ with $\Lambda=\bigcup_{i} \Lambda_{i}$ such that

$$
\left|\Pi_{\lambda}(A)\right| \leqq c_{i}(\lambda) p_{i}(A) \quad \text { for } \quad \lambda \in \Lambda_{i}
$$

where $c_{i} \in L_{2}(\Lambda, d \mu)$ and $p_{i}$ is a continuous seminorm on $\mathfrak{A}$.

(b) The completion of $\hat{\mathscr{D}}$ in the graph-topology is equivalent to

$$
\left\{\varphi \in L_{2} \mid\left(\lambda \mapsto \Pi_{\lambda}(A) \varphi(\lambda)\right) \in L_{2} \quad \text { for all } A \in \mathfrak{U}\right\}
$$

and this domain is a core for all $\hat{\Pi}(A), A=A^{*} \in \mathfrak{U}$.

(c) Restricted to $\mathscr{V}$ the linear functional $A \mapsto \Pi_{\lambda}(A)$ belongs to the closure of $\mathscr{Z}$ for almost all $\lambda$.

Proof. Since the proof is analogous to that of theorem 3.3 in [2] and theorem 4.1 we give only a sketch: By the usual spectral theorem we obtain the decomposition of the maximal abelian algebra $\mathscr{M}$ and of every single operator $\hat{\Pi}(A)$. Since $\mathscr{H}(\hat{\mathscr{D}})$ is separable by theorem 5.3 there is a countable set $\left\{\Omega_{i}\right\}$ of vectors in $\hat{\mathscr{D}}$ such that the linear span of $\bigcup_{i} \hat{\Pi}(\mathfrak{U}) \Omega_{i}$ is dense in $\mathscr{H}(\hat{\mathscr{D}})$. By multiplying with suitable characteristic functions (corresponding to projectors in $\hat{\mathscr{M}}$ ) we can arrange that the sets $\Lambda_{i}=\left\{\lambda \mid \Omega_{i}(\lambda) \neq 0\right\}$ are disjoint. Their union must be equal to $\Lambda$, up to a null set, since otherwise we could find a nonzero vector orthogonal to all $\hat{\Pi}(\mathfrak{U}) \Omega_{i}$. If we apply the nuclear spectral theorem to the maps $A \rightarrow \Pi(A) \Omega_{i} \rightarrow \Pi_{\lambda}(A) \Omega_{i}(\lambda)$ we get (a). (In order that $\Pi_{\lambda}(A)=\Omega_{i}(\lambda)^{-1}\left(\Pi(A) \Omega_{i}\right)(\lambda)$ is in $L_{2}$ we might have to change the measure on $\Lambda_{i}$ by multiplying it with $\left|\Omega_{i}(\lambda)\right|^{2}$ ). Statement (b) follows from the fact that every $\hat{\Pi}(A), A=A^{*}$ is essentially self adjoint on $\hat{\mathscr{D}}$ and (c) follows as in theorem 4.1.

\section{Applications to Quantum Field Theory}

In this section we discuss two applications of theorems 4.1 and 4.3. The first concerns Euclidean quantum field theory [2]. In this theory it is assumed that the Schwinger functions are the vacuum expectation values of a commutative field defined on Euclidean space-time. These functions define therefore a positive functional on the symmetric tensor algebra over the space of test functions for the Euclidean field operators. The usual test function spaces $\mathscr{S}$ and $\mathscr{D}$ satisfy all requirements for the application of theorem 4.3, which gives a necessary and sufficient condition for the Schwinger functions to be moments of a continuous cylinder measure on $\mathscr{S}^{\prime}$ or $\mathscr{D}^{\prime}$.

The second application is to Wightman theory. In [2] it was shown that there exist extremal Wightman states which do not correspond to field theories with a unique vacuum. We want to investigate under what conditions this can happen. As a starting point for the subsequent discussion we recall some results from [13]:

Let $\mathscr{A}$ denote the algebra of field operators of a tempered Wightman field defined on some domain $\mathscr{D}$ in a Hilbert space $\mathscr{H}$. Let $P_{0}$ be the projector on the vectors invariant under the translation group (and therefore also the Lorentz 
group) and define $\mathscr{H}_{0}=P_{0} \mathscr{H}$. We assume there is a cyclic set $\mathscr{Z}_{0} \subset \mathscr{H}_{0}$ of invariant vectors, i.e. such that $\mathscr{D}=$ linear span $\mathscr{A}_{\mathscr{Z}}$ is dense in $\mathscr{H}$. Let $\overline{\mathscr{D}}$ be the completion of $\mathscr{D}$ in the graph topology induced by $\mathscr{A}$ and define $\vartheta_{0}=\overline{\mathscr{D}} \cap \mathscr{H}_{0}$ and $\vartheta=$ lin. span $\mathscr{A} \vartheta_{0}$. With these notations the following theorem holds:

6.1. Theorem. (i) $P_{0} \vartheta \subset \vartheta_{0}$

(ii) The operators $A_{0}=P_{0} A P_{0}$ with $A \in \mathscr{A}$ generate a commutative algebra $\mathscr{A}_{0}$ on $\vartheta_{0}$.

Proof. See [13], Theorems 1 and 2.

The operators $A \in \mathscr{A}$ define a representation of the test function algebra $\mathscr{S}$ over Schwarz space [14], and the mapping $\mathscr{\mathscr { P }} \ni \underline{f} \rightarrow A(\underline{f}) \in \mathscr{A}$ is assumed to be strongly continuous, i.e. $f \rightarrow A(f) \varphi$ is a continuous map $\mathscr{\mathscr { S }} \rightarrow \mathscr{H}$ for all $\varphi \in \mathscr{D}$. Since it might not be obvious that the map $f \mapsto P_{0} A(f) P_{0} \varphi$ is continuous for $\varphi \in \vartheta$ (i.e. $P_{0} \varphi \in \overline{\mathscr{D}} \cap \mathscr{H}_{0}$ ), we make use of the following lemma:

6.2. Lemma. Suppose $\Pi$ is a strongly continuous *-representation of a barrelled *-algebra $\mathfrak{A}$ on a dense domain $\mathscr{D}_{\Pi}$ in a Hilbert space $\mathscr{H}_{\Pi}$. If $\hat{\Pi}$ is any extension of $\Pi$ to $a *$-representation of $\mathfrak{U}$ on domain $\mathscr{D}_{\hat{\Pi}} \subset \mathscr{H}_{\hat{\Pi}}=\mathscr{H}_{\Pi}$, then $\hat{\Pi}$ is also strongly continuous.

Proof. Since $\mathfrak{A}$ is barrelled, we have only to show that the absolutely convex, absorbing set

$$
\{A \mid\|\hat{\Pi}(A) \varphi\| \leqq 1\}
$$

is closed in $\mathfrak{A}$ for every $\varphi \in \mathscr{D}_{\hat{\Pi}}$. Now, since $\mathscr{D}_{\Pi}$ is dense in $\mathscr{H}_{\hat{\Pi}}$ we have

$$
\|\hat{\Pi}(A) \varphi\|=\sup _{\psi \in \mathscr{D}_{\Pi},\|\psi\|=1}|\langle\psi, \hat{\Pi}(A) \varphi\rangle| .
$$

If $A_{\alpha} \rightarrow A$ is a converging net in $\mathfrak{A}$ with $\left\|\hat{\Pi}\left(A_{\alpha}\right) \varphi\right\| \leqq 1$ for all $A_{\alpha}$, then also

$$
\left|\left\langle\psi, \hat{\Pi}\left(A_{\alpha}\right) \varphi\right\rangle\right|=\left|\left\langle\Pi\left(A_{\alpha}^{*}\right) \psi, \varphi\right\rangle\right| \leqq 1
$$

for all $\psi \in \mathscr{D}_{\Pi},\|\psi\| \leqq 1$, so

$$
|\langle\psi, \hat{\Pi}(A) \varphi\rangle|=\left|\left\langle\Pi\left(A^{*}\right) \psi, \varphi\right\rangle\right|=\lim _{\alpha}\left|\left\langle\Pi\left(A_{\alpha}^{*}\right) \psi, \varphi\right\rangle\right| \leqq 1
$$

Taking the supremum over $\psi$ yields $\|\Pi(A) \varphi\| \leqq 1$.

6.3. Corollary. If $\mathfrak{U}$ is a barrelled *-algebra, then the closure $\bar{\Pi}$ of a continuous *-representation $\Pi$ is also continuous.

Proof. This follows from 6.2 because the closure of a *-representation is also a *-representation, cf. [1] Lemma 2.6.

Because of this last result and Theorem 6.1 we may consider the algebra $\mathscr{A}_{0}$ as a continuous representation of the symmetric tensor algebra over $\mathscr{S}$. We define the topology of $\mathscr{A}_{0}$ as the corresponding quotient topology.

The decomposition theories of $\mathscr{A}$ and $\mathscr{A}_{0}$ are related by the following theorem, which is similar to theorem 3 in [13].

6.4. Theorem. (i) Every $b \in(\mathscr{A}, \vartheta)_{w}^{\prime}$ commutes with the representation of the Poincaré group and therefore with $P_{0}$.

(ii) Let $\mathscr{W}_{1}^{+}(\mathscr{A}, \vartheta)$ denote the order interval $0 \leqq b \leqq 1$ in the weak commutant $(\mathscr{A}, \vartheta)_{w}^{\prime}$ and let $\mathscr{P}_{0}$ denote the convex cone generated by elements of the form 
$P_{0} A\left(f^{*} \times f\right) P_{0}$ in $\mathscr{A}_{0}$. The restriction map

$$
b \mapsto b_{0}=b \mathscr{H}_{0}
$$

defines a homeomorphism (i.e. a topological bijection) $\mathscr{W}_{1}^{+}(\mathscr{A}, \vartheta) \rightarrow \mathscr{C}_{1}^{+}\left(\mathscr{A}_{0}, \mathscr{P}_{0}, \vartheta_{0}\right)$ where both sets are furnished with the weak operator topology.

Proof. For (i) we refer to [14] Theorem 4.

(ii) Suppose $b \in \mathscr{W}_{1}^{+}$. Since $b$ commutes with $P_{0}$ and weakly with $A, b$ commutes also weakly with $\mathscr{A}_{0}$. If $A_{0}=P_{0} A\left(f^{*} x f\right) P_{0}$, then $\left\langle\varphi, b A_{0} \varphi\right\rangle=\langle A(f) \varphi, b A(f) \varphi\rangle$ for $\varphi \in \vartheta_{0}$, so $b A_{0} \geqq 0$ and $(1-b) A_{0} \geqq 0$ on $\vartheta_{0}$. Moreover, $b$ is uniquely determined by its restriction to $\mathscr{H}_{0}$, for $\mathscr{Z}_{0} \subset \mathscr{H}_{0}$ is cyclic for $\mathscr{A}$ and therefore separating for the weak commutant. The map $b \mapsto b_{0}$ is weakly continuous and $\mathscr{W}_{1}^{+}$is weakly compact, so it remains only to show that every $b_{0} \in \mathscr{C}_{1}^{+}$is the restriction of some $b \in \mathscr{W}_{1}^{+}$. Now, the right hand side of

$$
\langle A(\underline{f}) \varphi|b| A(\underline{g}) \psi\rangle=\left\langle b_{0} \varphi, P_{0} A\left(\underline{f}^{*} \times \underline{g}\right) \psi\right\rangle
$$

is a well defined bilinear form in the vectors $A(f) \varphi$ and $A(g) \psi, \varphi, \psi \in \vartheta_{0}$. Indeed, it vanishes if $A(g) \psi=0$ and also if $A(f) \varphi=0$, because $b_{0}$ commutes with $\mathscr{A}_{0}$ so the right hand side is also equal to $\left\langle P_{0} A\left(g^{*}+f\right) \varphi, b_{0} \psi\right\rangle$. Both $b_{0}$ and $\left(1-b_{0}\right)$ are strongly positive with respect to $\mathscr{P}_{0}$. Therefore, the bilinear form is bounded by 0 and 1 so it is given by a bounded operator $b$ with $0 \leqq b \leqq 1$. By definition, this operator commutes weakly with $\mathscr{A}$, so it leaves $\mathscr{H}_{0}$ invariant. Finally, $b_{0}=b \nmid \mathscr{H}$ follows from the equality $\langle\varphi, b \psi\rangle=\left\langle\varphi, b_{0} \psi\right\rangle$ for $\varphi, \psi \in \vartheta_{0}$ because $\vartheta_{0}$ is dense in $\mathscr{H}_{0}$ according to theorem 6.1. (i).

6.5. Remark. Theorem 6.4 stays true if $\vartheta$ is replaced by $\mathscr{D}$ and $\vartheta_{0}$ by $\mathscr{D}_{0}:=$ lin. span $\mathscr{A}_{0} \mathscr{Z}_{0}$, because $\mathscr{D} \subset \vartheta \subset \mathscr{D}$, so $\mathscr{W}_{1}^{+}(\mathscr{A}, \vartheta)=\mathscr{W}_{1}^{+}(\mathscr{A}, \mathscr{D})$ and $\mathscr{C}_{1}^{+}\left(\mathscr{A}_{0}, \vartheta_{0}\right)=$ $\mathscr{C}_{1}^{+}\left(\mathscr{A}_{0}, \mathscr{D}_{0}\right)$.

It is not difficult to infer from Theorem 6.4 that the regular induced extensions $(\hat{\mathscr{A}}, \hat{\mathscr{M}}, \hat{\mathscr{D}})$ of $(\mathscr{A}, \mathscr{D})$ are in a one-to-one correspondence with regular $\mathscr{P}_{0}$-positive induced extensions $\left(\hat{\mathscr{A}}_{0}, \hat{\mathscr{M}}_{0}, \hat{\mathscr{D}}_{0}\right)$ of $\left(\mathscr{A}_{0}, \mathscr{D}_{0}\right)$ in the following sense: The Hilbert space $\mathscr{H}\left(\hat{\mathscr{D}}_{0}\right)$ is canonically embedded in $\mathscr{H}(\hat{\mathscr{D}})$ as the space of invariant vectors ${ }^{9}$ and the following diagram commutes:

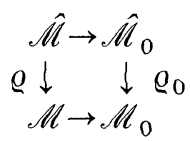

where the horizontal arrows stand for the restriction of the operator on $\mathscr{H}(\hat{\mathscr{D}})$ resp. $\mathscr{H}(\mathscr{D})$ to $\mathscr{H}\left(\hat{\mathscr{D}}_{0}\right)$ resp. $\mathscr{H}\left(\mathscr{D}_{0}\right)$ and $\varrho$ resp. $\varrho_{0}$ are the mappings $(\hat{\mathscr{A}}, \hat{\mathscr{D}})_{w}^{\prime} \rightarrow(\mathscr{A}, \mathscr{D})_{w}^{\prime}$ resp. $\left(\hat{\mathscr{A}}_{0}, \hat{\mathscr{D}}_{0}\right)_{w}^{\prime} \rightarrow\left(\mathscr{A}_{0}, \mathscr{D}_{0}\right)_{w}^{\prime}$ defined as in lemma 2.6. (i). The restriction maps are ultraweakly continuous isomorphisms and since the extensions are regular, also the vertical arrows stand for bijective mappings which are homeomorphisms for the weak topology when restricted to the unit balls $\hat{\mathscr{M}}_{1}$ resp. $\left(\hat{\mathscr{M}}_{0}\right)_{1}$. We do not

9 i.e. invariant under the extended representation $\hat{\mathscr{U}}$ of the Poincaré group on $\mathscr{H}(\hat{\mathscr{D}})$ which is defined in the following way: If $g \rightarrow U(g)$ is the representation on $\mathscr{H}(\mathscr{D})$, then $\hat{\mathscr{U}}(g) \hat{m} \varphi:=\hat{m} U(g) \varphi$ for $\hat{m} \in \hat{\mathscr{M}}, \varphi \in \mathscr{D}$. 
write down a formal proof of these statements, since they are not essential for theorem 6.6.

Since we want to apply the results of section 4 we now assume there is at least one cyclic vector $\Omega \in \mathscr{H}_{0}$ and define $\mathscr{D}=\mathscr{A} \Omega$ and $\mathscr{D}_{0}=\mathscr{A}_{0} \Omega$. Let $W$ denote the Wightman state defined by $\Omega$ and $W_{0}$ the corresponding state on $\mathscr{A}_{0}$.

6.6. Theorem. The following statements are equivalent:

(i) $W$ has a weak integral decomposition

$$
W=\int_{\Lambda} W_{\lambda} d \mu_{\lambda}
$$

with $\Lambda, d \mu$ as in theorem 4.1, and $W_{\lambda}$ a Wightman state with cluster property for almost all $\lambda$.

(ii) $W_{0}$ has a weak integral decomposition

$$
W_{0}=\int_{\Lambda} W_{0 \lambda} d \mu_{\lambda}
$$

$\Lambda, d \mu$ as above and $W_{0 \lambda}$ a $\mathscr{P}_{0}$-positive character for almost all $\lambda$.

(iii) $W_{0}$ is positive on $\mathscr{P}\left(\mathscr{P}_{0}^{\prime+}\right)$, defined as in Lemma 4.4 .

Proof. The equivalence of (ii) and (iii) follows from Theorem 4.1. The equivalence of (i) and (ii) should be fairly clear from the consideration above, but can also be shown directly as follows: Given a decomposition of $W_{0}$ into $\mathscr{P}_{0}$-positive character we define

$$
W_{\lambda}(f)=W_{0 \lambda}\left(A_{0}(\underline{f})\right) .
$$

$W_{\lambda}$ is a positive linear functional and $\int W_{\lambda}(f) d \mu_{\lambda}=W_{0}\left(A_{0}(\underline{f})\right)=W(\underline{f})$. Every decomposition of $W$ into states is a decomposition into Wightman states, so we have only to check the cluster property. For any $f, g$ we have

$$
W_{\lambda}(f) W_{\lambda}(\underline{g})=W_{0 \lambda}\left(A_{0}(f)\right) W_{0 \lambda}\left(A_{0}(g)\right)=W_{0 \lambda}\left(A_{0}(f) A_{0}(\underline{g})\right)
$$

because $W_{0 \lambda}$ is a character on $\mathscr{A}_{0}$. Let $\lambda \rightarrow m(\lambda)$ be any bounded measurable function on $\Lambda$ corresponding to an operator $m \in(\mathscr{A}, \mathscr{D})_{w}^{\prime}$.If $a \in \mathbb{R}^{4}$ is any translation, we have

$$
\begin{aligned}
\int\left\{W_{\lambda}\left(f \times \underline{g}_{\{a\}}\right)-W_{\lambda}(f) W_{\lambda}(g)\right\} m(\lambda) d \mu_{\lambda}= & \left\langle A\left(f^{*}\right) \Omega, m A\left(g_{\{a\}}\right) \Omega\right\rangle \\
& -\left\langle A_{0}\left(\underline{f}^{*}\right) \Omega, m A_{0}(g) \Omega\right\rangle .
\end{aligned}
$$

Since weak $\lim _{a \rightarrow \infty} A\left(g_{\{a\}}\right) \Omega=A_{0}(g) \Omega$ and $m$ commutes with $P_{0}$, this vanishes for all $m$. By the estimate (ii) (c) in Theorem 4.1 and since

$$
\left|W_{\lambda}\left(f \times \underline{g}_{\{a\}}\right)\right| \leqq W_{\lambda}\left(f^{*} \times \underline{f}\right)^{1 / 2} \cdot W_{\lambda}\left(g^{*} \times g\right)^{1 / 2},
$$

we may interchange limes and integral and obtain

$$
\lim _{a \rightarrow \infty} W_{\lambda}\left(\underline{f} \times \underline{g}_{\{a\}}\right)=W_{\lambda}(\underline{f}) W_{\lambda}(g) \quad \text { a.e. }
$$

To get a null set independent of $f$ and $g$ we appeal to separability (or nuclearity) of $\mathscr{S}$.

The other direction (i) $\Rightarrow$ (ii) is obvious since a decomposition of $W$ into clustering states implies a diagonalization of $P_{0}$. 


\title{
References
}

1. Powers, R.T.: Commun. math. Phys. 21, 85-124 (1971)

2. Borchers, H.J., Yngvason, J.: On the algebra of field operators. The Weak Commutant and Integral Decomposition of states. Commun. math. Phys. 42, 231-252 (1975)

3. Shohat, J.A., Tamarkin, J.D.: The Problem of Moments, Surveys No. 1, Amer. Math. Soc. New York 1943

4. Choquet, G.: Lectures on Analysis, Vol. II, New York: Benjamin 1969

5. Berezansky,Ju.M.: Generalized Power moment problems. In: Hilbert space operators and operator algebras, Sz-Nagy, B. (ed.), Amsterdam-London: North Holland 1972

6. Powers, R.T.: Transactions Amer. Mat. Soc. 187, 261-293 (1974)

7. Lassner, G.: Rep. math. Phys. 3, 279--293 (1972)

8. Dixmier,J.: Les algèbres d'operateurs dans l'espace Hilbertien, 2ième édition. Paris: GauthierVillars 1969

9. Maurin, K.: Generalized Eigenfunction Expansion and Unitary Representations of Topological Groups. Warszawa: Polish Scientific Publishers 1968

10. Gel'fand, I. M., Vilenkin, N. Ya.: Generalized functions, Vol. 4. New York: Academic Press 1968

11. Pietsch, A.: Nuclear locally convex spaces, Berlin-Heidelberg-New York: Springer 1972

12. Symanzik, K.: Euclidean Quantum Field Theory. In: Proc. of the International School of Physics

"ENRICO FERMI", Varenna Course XLV, ed. R. Jost, New York: Academic Press 1969

13. Borchers, H. J.: Commun. math. Phys. 1, 49-56 (1965)

14. Borchers, H.J.: Nuovo Cimento 24, $1118-1140$ (1962)

Communicated by H. Araki

\author{
H. J. Borchers \\ J. Yngvason \\ Institut für \\ Theoretische Physik \\ Universität Göttingen \\ D-3400 Göttingen \\ Bunsenstraße 9 \\ Federal Republic of Germany
}


